\title{
The Role of Current College Students Physical Training Camp in Physical Education of Colleges
}

\author{
Jie Wang \\ University of Electronic Science and Technology of China, Chengdu, Sichuan, 610054
}

Keywords: sports education camp, college physical education, teaching role

\begin{abstract}
With the continuous reform of college sports, the continual innovation and development of sports training in higher education has also been carried out. This paper analyzes the role of college sports training camp in physical education and proves that the college sports training camp has the following characteristics, playing an important role in promoting the cultivation of qualified personnel.
\end{abstract}

\section{Introduction}

In order to respond to the call of the country, all colleges have joined different types and levels of college sports training camps in physical education, which also resulted in the transformational optimization and upgrading of grass-roots sports organizations. Due to the innovative teaching method, not only the traditional rigid teaching mode has been changed, but also the educational circles have been praised.

\section{The formation and development of college sports training camp}

With the promulgation of the national goal of new education, the major colleges have also responded positively and adopted a variety of ways of reform, which just entered the university students, as soon as they enter the school will face the following aspects Question: Students in self-evaluation, learning, interpersonal aspects of life adaptations may exist some of the physical or psychological problems [1]. In order to relieve the pressure of students and enjoy a good college life, sports training organizations that can provide students with the ability to practice and solve problems have come into being.

University sports training camp is a university physical education teachers and extracurricular sports enthusiasts together, the use of leisure time for sports training and health activities. Is a new teaching mode, all members are voluntarily joined. Not only physical training camps, basketball, volleyball, soccer, tennis, taekwondo and other traditional training camps have been established, but some training camps for expanding programs have also been set up after careful examination according to the opinions of all community staff. In response to the needs of the majority of college students, our camp also combines social, entertainment, fitness, will exercise and development potential.

University sports training camp is developed after college sports club, which can not only promote the organizational form and management mechanism of college sports clubs, but also make the university physical education more suitable for the needs of modern society to train talents [2]. University sports training camp is mainly for winter and summer vacations and after-hours set up, will not take up student learning and participation in social practice, its development can not only improve college students enthusiasm for sports activities, but also to promote the extensive development of college sports clubs, For the quality of innovative personnel provided a guarantee.

\section{The role of college sports training camp in college physical education}

Starting from the integrity of university education, applying sports training camp to university physical education is beneficial to expanding the students' understanding of physical education 
knowledge. At the same time, more university students can conduct scientific and healthy physical exercise and form lifelong physical education and lifelong health Consciousness and habits. Standing on the perspective of individual students, sports training camps can help college students to establish a correct world outlook, outlook on life and values, which can not only enhance their physical functions, but also enhance their mental health index and constantly improve their personalities.

In detail, adding a socialized and industrialized sports training camp to physical education at colleges can allow students to increase their internal reflection on their own, to reflect on their psychological inertia, to have an objective and fair evaluation of themselves and to improve themselves Binding, at the same time by participating in such activities can also exercise their communicative competence, through communication and coordination and cooperation to improve their practical ability, with scientific guidance, I believe this kind of physical exercise will be able to achieve a multiplier effect. Carrying forward the competitive spirit of sports culture has a very big promotion effect on the social moral standards of college students and the improvement of sports aesthetic ability.

In order to give full play to the role of sports training camp, to promote the development of university sports activities, enrich the campus sports culture, improve the training level of college sports athletes reserve personnel, college sports training camp to use promotional boards to increase students' fitness knowledge. During the period of formation, university sports training camps should hold regular fitness knowledge lectures to enhance students' understanding of sports activities, and clarify the conditions, purposes and requirements for participating in college sports training camps. From the screening of applicants, only real college students with physical training qualifications can join, and we also need to start the test, such as the applicants to do 50 push-ups on site or to show his good sports, through him in the Site performance to determine whether to have the qualifications to join. We do not have any gender, nationality or race restrictions on applicants, but we also do not recruit students who have heart disease or other physical disabilities. You may not have any basis for physical activity, but you must ensure that your body is healthy. . Undergraduates' physical training camps can enhance the students' ability of scientifically exercising under self-supervision, expand the space for college students to participate in physical fitness activities, create a strong sports culture atmosphere on campus, active students' sports and cultural life, and cultivate their awareness of lifelong sports.

The purpose of physical education reform in colleges is to give full play to the functions and functions of physical education and enhance students 'physique and health, which can not only improve students' health but also promote their all-round development [3] University sports training camp should give full play to students' dominant position, take students as the center, and use innovative means and methods to stimulate their interest in sports study. The goal of sports training and content selection should be consistent, follow the concept of health first to carry out the construction.

The combination of extracurricular time with the time in class is an effective way to expand the time and space for students to participate in physical activities. Our training is not limited to indoor sports training. Projects like rock climbing, climbing, cliff falling, field camping, orienteering, streams, exploration and so on are all accessible to our training camps. We can improve the physical fitness and technical skills of students by organizing activities and competitions. College sports training camp should use the theory with practical methods, taking full account of the actual situation of members of the team after the targeted training. It has the following characteristics: give full play to the student's dominant position, demonstrate the individualized development of students, have outstanding professional training of physical education teachers, I believe we can maximize the potential of students, challenge the limits of self, maximize students' physical and Psychological adaptability.

Sports training camp for college students can achieve the goal aspirations of sports fans. Sports fans can not only enjoy the joy of sports, but also gain the satisfaction of success, increase their self-confidence, fully demonstrate their personal charisma, and establish the correct values of 
outlook on life.

The college student physical training camp is helpful to improve students' social adaptability and mental health level, and then enhances their own viability. Under the guidance of teachers 'guidance and team members' training, college sports training camps receive direct knowledge through full participation of individuals in activities. We take a step-by-step training method from simple physical training to survival training, The final upgrade to the psychological, personality, and even management training. For example, our field training camps and physical training camps expand the training is the use of mountains, rivers, woods and other natural landscape, through the careful design of our trainers, both to achieve the temper, cultivate sentiments, improve personality, develop solidarity, we can also think about the cognitive, emotional, volitional and communicative activities. We have specific operational process design, which requires students to train in accordance with the formal operating procedures and must devote themselves to challenging their own physiology as well as the psychological limit [4]. Such group action is more about the ability to cultivate solidarity and cooperation so that the participating students can enhance the team's sense of honor and disgrace, make unremitting efforts for collective honor, enhance the sense of teamwork, triumph and pride, and gain a rare life Experience [5], improve college students' social adaptability and mental health level, enhance the self-survival ability of college students.

\section{Application of sports training camp in the university}

Sports training camp to establish a diversified operating mechanism. Students who join the training camp on their own initiative and in accordance with the principle of voluntariness must participate in sports training in accordance with the rules and regulations and fulfill their obligation to exercise. In order to demonstrate the flexibility of the curriculum, we must establish a diversified operating mechanism that adapts to the teaching mode of college sports training camp to stimulate students 'interest in and enthusiasm for more active participation in the teaching activities of physical training camp, to tap and develop the potential of college students' Cultivate college students lifelong sports awareness and will. University life itself is full of a variety of exciting activities, our training camp to get the favor of the majority of students need to constantly innovate the content and methods of training, physical education will be promoted to industrialization, social teaching mode, let college students in the use of extra-curricular sports training camp to achieve institutional learning and social practice.

For example, we can use publicity boards and flyers to expand publicity and enrollment. We can also give students an intuitive feeling about the course content and training methods of training camps by means of lectures. At the same time, they adopt experiential teaching methods to pass Students' personal experiences increase their real feelings and thus attract more students to join. This high degree of freedom and flexibility of the enrollment mechanism can help students fully aware of the difference between training camp and traditional physical education.

Establish a new type of teacher-student relationship. Not only on the book, nor is not a book is not a teacher, respect for student autonomy and self-esteem, equality, respect and understanding based on the establishment of cooperative relations. While cultivating students 'autonomy, we must also give proper guidance and strive to create a relatively relaxed and free teaching environment and cultivate the students' courage to express their opinions.

\section{Training content of college sports training camp}

Body balance depends mainly on the strength of the core parts of the stability of the central nervous system by the body conduction, ontology feel their participation and reflected in the mechanism of action control. We can use simple equipment for muscle strength exercises such as speed ball, Roman chair, flat stool, Swiss ball, supine board, balance pad, etc., to abandon the past with external force to support the body exercises, with a more scientific way of body exercises.

For example, the athlete sits on a Swiss ball, supports the ball with both hands to maintain balance, and simultaneously uses the elastic band to pull the thigh. The left leg of the athlete 
benefits from the balance pad, the lower position fixes the elastic band, balances the right leg and knees, puts the arm straight on both sides of the body, holds the handle of the elastic band with both hands, the body transfers the elastic band backward in a tight state, slowly pull the stretch belt before, so that the elastic belt and arms are linear, still for a while and then restore.

Weight training must master the body can carry the load, to avoid being too hard to hurt the situation. Appropriate mechanical weight-lifting exercises can enhance the athlete's muscle energy, such as holding a dumbbell for weight-bearing exercises: the athlete assumes a supine position, presses the middle of the speed ball against his lumbar spine, lifts his legs and maintains a $90^{\circ}$ flexion, Hands holding dumbbells for practice.

Unbalanced start double training, is conducive to the overall control of the movement and explosive power. Specific ways are as follows: two athletes face to face on the two Bo speed ball, open legs and shoulder level, the two were cross-handshake form, while squat exercises, in order to maintain balance, both will force each other until one of them can't keep balance and fall from the speed ball. This repeated practice, you can increase the balance of athletes.

\section{Recommendations for colleges to establish college sports training camp}

School leaders should raise their awareness of the importance of physical exercise, support and encourage students' participation in sports training camps, and provide human, material and financial resources when necessary.

The effective management of the operating mechanism of college sports training camp to make the system more complete and effective;

Schools should strengthen their safety precautions, emergency preparedness plans.

Regular training of trainers in sports training camp, trainers have the ability to deal with simple sports injuries.

The construction of the medical office of the school must establish a green channel for the sports training camp. Injuries of the athletes are more serious and urgent under normal circumstances. The medical care of the medical staff must be kept up to the standard.

Schools should regularly organize suitable sports activities for the athletes, such as marathon competitions and extreme sports activities to enhance students' sense of participation and increase their emphasis on them.

\section{Conclusions}

The physical training camp for students of communication skills, cooperation has improved, can use the correct method to overcome the psychological inertia, a more comprehensive understanding of themselves, access to more learning opportunities, we resist the spirit of pollution, to prevent and correct bad an important measure of behavior.

\section{References}

[1] Wang Xiaohong. The Role of College Students Physical Education Camp in Physical Education in Colleges [J]. Journal of Wuhan University of Technology, 2009, 31 (08): 80-82.

[2] Liu Qiang, Zou Yanning, Yang Huan. Application of college sports training camp in university physical education [J]. Journal of Physical Education (2016), 2016, (08): $156+158$.

[3] Huang Q. Exploration of ways to cultivate interest in college physical education [J]. Contemporary Sports Science and Technology, 2016, 6 (33): 51-52.

[4] Liu Qiang, Wang Zupeng. Application of college physical education camp training in physical education [J]. Sports World (Academic Edition), 2016, (12): 112.

[5] Liang Yi. Study on the Significance and Development Principles of College Physical Training Innovation [J]. Contemporary Sports Science and Technology, 2017, 7 (17): 47 + 49 